\title{
Postoperative Pain in Endodontically Treated Teeth: A Cross-sectional Study of Retrospective Data
}

\author{
Bárbara S Fornari ${ }^{1}$, Caroline Solda ${ }^{2}$, Lilian Rigo ${ }^{3}$
}

\begin{abstract}
Aim: This study aimed to assess the occurrence of postoperative pain and associated factors in patients treated endodontically at a Postgraduate Center in Endodontics in Southern Brazil.

Materials and methods: The evaluation was performed using the medical records of 658 patients. Pulp conditions, postoperative pain, pain intensity, edema, number of sessions (single or multiple), and medication administration in the postoperative period were analyzed. For data analysis, descriptive analyses and univariate and multiple regressions were performed. In the multiple analyses, odds ratios (OR) and their respective $95 \%$ confidence intervals were estimated, crude and adjusted for exposure variables in a binary logistic regression model ( $p$-value $<0.05)$.

Results: To perform the adjusted logistic regression, all variables associated with $p$-value <0.10: gender, edema, and pulp condition (bio- and necropulpectomy) entered the crude model. After the multivariate analysis, a statistically significant association was found between the outcome variable of the presence of postoperative pain and the independent variable of pulp condition, and the presence of pain was associated with patients who underwent endodontics on teeth with live pulp and edema with greater chances of postoperative pain.

Conclusion: It was concluded that the pulp condition and edema affected postoperative pain.

Clinical significance: The occurrence of postoperative pain was around 30\% in both single and multiple sessions, and it was considered relevant for the dental clinic. Pulp condition affected postoperative pain, and the presence of pain was associated with patients who underwent endodontics on teeth with live pulp.

Keywords: Dental pulp, Dental pulp necrosis, Edema, Endodontics, Pain.

The Journal of Contemporary Dental Practice (2021): 10.5005/jp-journals-10024-3021
\end{abstract}

\section{INTRODUCTION}

Postoperative pain in patients subjected to endodontic treatment is common; it creates discomfort for patients and concerns professionals. The onset of postoperative pain is usually due to the acute inflammatory response in periradicular tissues, starting within a few hours or days after the endodontic treatment. ${ }^{1}$ Postoperative pain may be associated with the number of consultations, preoperative factors, transoperative complications, the extension of the periapical lesion, quality of the coronary sealing, inadequate instrumentation, intracanal medication extrusion, extrusion of debris, and apical irrigators. ${ }^{2}$

The presence of pain and/or edema after endodontic treatment is called flare-up, which has multifactorial etiology and may be of either mechanical origin or microbial contamination during the endodontic procedure. ${ }^{3}$

Postoperative pain or flare-up may indicate a clear relationship with the pulp condition even after successful endodontic therapy. ${ }^{4}$ The purpose of root canal treatment is to eliminate the microbial load, promote periradicular tissue healing, and prevent microbial recolonization. ${ }^{5}$

The mechanical instrumentation and root canal disinfection have been simplified, which makes the single consultation treatment a more practical and acceptable treatment regime than multiple consultations. ${ }^{2}$

During root canal debridement, all instrumentation techniques with manual or rotary instruments produce an apical extrusion of debris even when preparation occurs at the apical end, causing periapical inflammation known as outbreaks, so
${ }^{1-3}$ Department of Dentistry, Meridional Faculty/IMED, Passo Fundo, Rio Grande Do Sul, Brazil

Corresponding Author: Lilian Rigo, Department of Dentistry, Meridional Faculty/IMED, Passo Fundo, Rio Grande Do Sul, Brazil, Phone: +55 54 999270441, e-mail: lilian.rigo@imed.edu.br

How to cite this article: Fornari BS, Solda C, Rigo L. Postoperative Pain in Endodontically Treated Teeth: A Cross-sectional Study of Retrospective Data. J Contemp Dent Pract 2021;22(1):42-46.

Source of support: Nil

Conflict of interest: None

postoperative pain is also related to mechanical and chemical lesions, performed in either single or multiple consultations and regardless of the instrumentation technique. ${ }^{6}$ Thus, important advances in rotary instrumentation have led to the introduction of numerous systems with innovative designs in recent years. However, all the preparation techniques and instruments available to date are still associated with some degree of extrusion of debris. $^{7}$

Endodontic treatment has recently undergone several modifications, such as the type of irrigating substance used, apical preparation limit, root canal instrumentation technique, and root canal filling technique, and postoperative pain is still reported by patients and dentists.

Understanding the causes related to pain after endodontic treatment is of great importance for professionals to prevent or manage this undesirable condition. 
Therefore, the present study aimed to assess the presence of postoperative pain and associated factors in patients with endodontically treated teeth in a Postgraduate Center in southern Brazil.

\section{Materials and Methods}

The research was submitted and approved by the Ethics and Research Committee under number CAAE 0011.0.362.000-10, according to Resolution 466/2012.

\section{Study Design and Sample}

The research had a quantitative approach with a cross-sectional design, using retrospective data from 658 medical records of patients with teeth submitted to endodontic procedures in an Endodontic Postgraduate Center in the city of Passo Fundo, RS, Brazil, in 2016, 2017, and 2018. Endodontic treatments were carried out by students specialized in endodontics and graduate studies, with help and support from professors with doctorate in endodontics.

The inclusion criteria were all patients aged 18 years or older who had only one endodontically treated tooth between 2016 and 2018 , regardless of the type of tooth, excluding those subjected to endodontic treatment in two or more dental elements. Thus, all 658 medical records of patients with these conditions were included in the study according to the criteria.

The protocol used for the treatment of root canal is standard for all students at the Postgraduate Center, with hybridization of movements (rotary and reciprocating). After clinical and radiographic diagnoses, anesthesia and approach are performed, followed by exploration, catheterization, or disinfectant penetration of the root canals with manual files of diameter $\# 08$, \#10, and \#15. The root canal disinfection protocol is with $2 \%$ chlorhexidine gel (deposited in the root canal) and saline solution (1.5-ml syringe per canal for each instrument change). After using file \#15, the cervical third of the teeth were prepared with the Wave One Gold 20/07 file in thinner roots or the Wave One Gold Large 45/05 in broader roots as in the upper anterior ones. Next, the apical locator and digital radiography are used to define the actual working length of the tooth treated. Thus, the Wave One Gold instrument used later is again inserted into the canal with brushing movements along the entire length of the tooth up to foramen zero. Then, the rotating files Hero \#30/02, Hero \#40/02, and Hero \#45/02 are used in the rotational movement along the entire length of the tooth up to foramen zero. After instrumentation, the final irrigation protocol is carried out, applying 17\% EDTA for 3 minutes in the root canal, subsequently irrigated with saline solution. The root canal is filled using hydraulic compression with the Odous de Deus gutta-percha cones, in which the proof of the cone must be $2 \mathrm{~mm}$ short of the apex. The endodontic cement used is the AH Plus. In cases of multiple consultations, the intracanal medication (ICM) used is $2 \%$ chlorhexidine gel in vital pulps and calcium hydroxide (UltraCal) in necrotic pulps.

At the end of endodontic treatment, patients were scheduled to return after one month of treatment for a reassessment. In this return, using a clinical file for postoperative control attached to the medical record of each patient, it was verified whether there was pain after the endodontic treatment.

The data listed on the form for this study were gender (male/ female), tooth (incisors/canines/premolars/molars), pulp condition (biopulpectomy/pulp necrosis or retreatment), treatment sessions (single or multiple), pain after endodontic treatment (yes/no), and duration. The scale to assess pain intensity was from 1 to 4 (tolerable pain) and 5 to 10 (unbearable pain); postoperative medicationanalgesic, anti-inflammatory, or antibiotic-(yes/no), and the presence of edema in the region (yes/no).

\section{Data Collection and Analysis}

For data collection, a database was built in the Excel software to store the information in the medical records of each patient, according to the variables aforementioned: gender, tooth, pulp condition, treatment sessions, postendodontic medication, presence of postoperative pain, pain intensity, and edema.

After that, the data obtained from the medical records of the patients assisted were exported to the IBM SPSS ${ }^{\mathrm{TM}}$ statistical software (Statistical Package for the Social Sciences), version 20.0, Armonk, New York. The statistical analysis consisted of a descriptive data analysis and the subsequent application of a specific test to verify the potential associated factors.

For data analysis, descriptive analyses and univariate and multiple regressions were performed. In the multiple analysis, odds ratios (OR) and their respective $95 \%$ confidence intervals were estimated, crude and adjusted for exposure variables in a binary logistic regression model ( $p$-value $<0.05$ ).

\section{Results}

The total number of the samples registered in the medical records was 658 teeth from individuals, and the majority were women (62.8\%). Table 1 shows the descriptive data for all the teeth analyzed,

Table 1: Descriptions of the frequency of data in the medical records of patients treated endodontically at a Postgraduate Center, RS, Brazil, 2019

\begin{tabular}{|c|c|c|}
\hline Variables & $N(658)$ & $\%(100)$ \\
\hline \multicolumn{3}{|l|}{ Gender } \\
\hline Male & 245 & 37.2 \\
\hline Female & 413 & 62.8 \\
\hline \multicolumn{3}{|l|}{ Teeth } \\
\hline Canines and incisors & 113 & 17.2 \\
\hline Premolars & 211 & 32.1 \\
\hline Molars & 334 & 50.8 \\
\hline \multicolumn{3}{|l|}{ Pulp condition } \\
\hline Living pulp & 274 & 41.6 \\
\hline Pulp necrosis & 351 & 53.3 \\
\hline \multicolumn{3}{|l|}{ Treatment session } \\
\hline Single & 520 & 79.0 \\
\hline Multiple & 138 & 21.0 \\
\hline \multicolumn{3}{|l|}{ Pain } \\
\hline Yes & 199 & 30.2 \\
\hline No & 459 & 69.8 \\
\hline \multicolumn{3}{|l|}{ Edema } \\
\hline No & 619 & 94.1 \\
\hline Yes & 39 & 5.9 \\
\hline \multicolumn{3}{|l|}{ Pain intensity } \\
\hline Tolerable 1-4 & 94 & 14.3 \\
\hline Unbearable 5-10 & 105 & 16.0 \\
\hline No & 459 & 69.8 \\
\hline \multicolumn{3}{|c|}{ Postoperative medication } \\
\hline Yes & 207 & 31.5 \\
\hline No & 451 & 68.5 \\
\hline
\end{tabular}


Table 2: Univariate (crude) and multivariate (adjusted) binary logistic regression model for the variable of postoperative pain, 2019

\begin{tabular}{|c|c|c|c|c|}
\hline & $\begin{array}{l}\text { Crude OR } \\
(95 \% \mathrm{Cl})\end{array}$ & $p$-value & $\begin{array}{l}\text { Adjusted OR } \\
(95 \% \mathrm{Cl})\end{array}$ & $p$-value ${ }^{*}$ \\
\hline $\begin{array}{l}\text { Gender } \\
\text { Male } \\
\text { Female }\end{array}$ & $\begin{array}{l}1 \\
1.41 \\
(0.99-2.01)\end{array}$ & 0.052 & $\begin{array}{l}1.38 \\
(0.96-1.98)\end{array}$ & 0.078 \\
\hline $\begin{array}{l}\text { Groups of teeth } \\
\text { Maxillary teeth } \\
\text { Mandibular teeth }\end{array}$ & $\begin{array}{l}1 \\
1.09 \\
(0.78-1.52)\end{array}$ & 0.607 & - & - \\
\hline $\begin{array}{l}\text { Pulp condition } \\
\text { Necropulpectomy } \\
\text { Biopulpectomy }\end{array}$ & $\begin{array}{l}1 \\
1.51 \\
(1.08-2.11)\end{array}$ & 0.015 & $\begin{array}{l}1.60 \\
(1.13-2.26)\end{array}$ & 0.007 \\
\hline $\begin{array}{l}\text { Edema } \\
\text { No } \\
\text { Yes }\end{array}$ & $\begin{array}{l}1 \\
4.56 \\
(2.32-8.99)\end{array}$ & $<0.000$ & $\begin{array}{l}1 \\
5.01 \\
(2.51-9.97)\end{array}$ & $<0.001$ \\
\hline $\begin{array}{l}\text { Treatment sessions } \\
\text { Single } \\
\text { Multiple }\end{array}$ & $\begin{array}{l}1 \\
0.81 \\
(053-1.23)\end{array}$ & 0.324 & - & - \\
\hline
\end{tabular}

Adjusted by the variables of gender, groups of teeth, pulp condition, edema, and number of treatment sessions $(p<0.05)$; *Wald test $(p<0.05-$ statistically significant); OR, odds ratio; $95 \% \mathrm{Cl}-95 \%$ confidence interval; $\%$, frequency-percentage

noting that $53.3 \%$ were diagnosed with pulp necrosis and $79 \%$ were treated in a single consultation. The prevalence of postoperative pain was $30.2 \%$.

To perform the adjusted logistic regression, all the variables associated with $\mathrm{p}$-value <0.10: gender, edema, and pulp condition (bio- and necropulpectomy) entered the crude model. The variables of number of endodontic treatment sessions (single or multiple) and groups of teeth (upper and lower) did not enter the final model. However, after the multivariate adjustment, the variables of pulp condition and having had edema remained significant $(p<0.05)$, and gender lost its association with the final adjusted model. The patients subjected to biopulpectomy are 1.60 (95\% Cl 1.13-2.26) times more likely to have postoperative pain, as those who had edema are 5.01 times more likely to have pain after the endodontic treatment (95\% Cl 2.51-9.97), according to Table 2.

\section{Discussion}

Apical extrusion by infected debris, changes in the root canal microbiota, incomplete mechanical instrumentation, and secondary infections are often causes of postoperative pain. Some studies that relate the number of consultations and irrigation solutions to pain as well as the diagnosis 8,9 reported that endodontic treatment is based on two fundamental principles: mastery of anatomy and infection control, carried out through access to the pulp chamber, the main canals, dentinal tubules, and branches, allowing cleaning and disinfecting the root canal system.

In the present study, the prevalence of postoperative pain was assessed in all patient reconsultation forms in the years of 2016, 2017, and 2018 involving necrotic and vital teeth and cases of endodontic retreatments performed in single and multiple consultations in patients of the Postgraduate Unit of Faculdade Meridional/IMED.
It was verified, using patients' reports in the treatment records, whether they presented pain or not after the root canal treatment sessions or session.

In this study, one of the ways of evaluating postoperative pain was through a pain intensity scale in the postoperative medical record, with 1 to 4 being tolerable and 5 to 10 being unbearable. A rate of $14.3 \%$ reported that pain was tolerable and $16.0 \%$ reported that postoperative pain was unbearable. In the study by Kherlakian et al., ${ }^{7}$ patients were asked to rate the intensity of postoperative pain in a visual analog scale according to four classes (no pain, mild pain, moderate pain, and severe pain) after 24 hours, 48 hours, 72 hours, and 7 days. In the present study, the highest postoperative pain scores were observed 24 hours after the treatment in all instrumentation groups, with a significant decline thereafter. No patient reported severe pain, and all were being medicated in the postoperative period.

In this study, the prevalence of postoperative pain was higher in patients subjected to biopulpectomy treatment 1.60 (95\% Cl 1.132.26), as well as in the study by Segura-Egea et al., ${ }^{10}$ which determined that the prevalence of postoperative pain was significantly higher in teeth with irreversible pulpitis (biopulpectomy) and acute apical periodontitis compared to the group with necrotic pulp and chronic apical periodontitis. According to Silva et al., ${ }^{6}$ during canal debridement, all instrumentation techniques with manual or rotary instruments produce the apical extrusion of debris even when the preparation takes place at the apical end, causing periapical inflammation known as outbreaks; thus, postoperative pain is also related to mechanical and chemical lesions made in either single or multiple consultations and regardless of the instrumentation technique.

Some authors attribute the extrusion of debris as one of the causes of postoperative pain, which may be related to the type of system and kinematics used. Recent systems were testedProTaper Next (PTN) rotary system; WaveOne alternative system, Twisted File Adaptive rotating/alternative system, and SelfAdjusting File (SAF) system. The extrusion of apical debris was displayed at some level. ${ }^{11}$

Some authors suggest that rotational kinematics may be associated with lower extrusion of debris than reciprocal instrumentation. ${ }^{12}$ The present study used the hybrid movement technique, that is, reciprocal and rotational kinematics.

The data from the literature present a variation of results regarding the diagnosis with postoperative pain. In the present study, postoperative pain was more present in the cases of biopulpectomy. This contrasts the studies by Hameed et al., ${ }^{1}$ Onay et al., ${ }^{13}$ and Baker et al., ${ }^{14}$ in which postoperative pain was more frequent in the cases of necropulpectomy. The study by Direnzo et al., ${ }^{15}$ in turn, did not show difference between the treatments performed in vital and nonvital teeth.

There was no significant difference concerning postoperative pain performed in conventional endodontic treatment in a single consultation or multiple consultations. The study by Rigo et al., ${ }^{16}$ also carried out at the same Postgraduate Center, found that postoperative pain was associated with patients who performed a single session. In a study by lqbal et al., ${ }^{17}$ there was no statistically significant difference between one and two visits and the presence of periapical lesion was the most important factor associated with postoperative pain. In research by Hameed et al. ${ }^{1}$ and Direnzo et al., ${ }^{15}$ there were no significant differences between postoperative pain and endodontic treatment in single or multiple consultations. The study by Hepsenoglu et al. ${ }^{2}$ showed that 
endodontic retreatment from a single visit had a lower incidence of postoperative pain.

Yaylali et al. ${ }^{18}$ assessed the maintenance of apical patency concerning postoperative pain and observed that foraminal enlargement did not increase postoperative pain in teeth with necrotic pulp and did not increase postoperative pain in teeth with vital pulp. Moreover, maintaining apical patency did not increase postoperative pain when a single-canal or two-visit treatment approach was used. Another study on the relationship between apical patency and postoperative pain by Abdulrab et al. ${ }^{19}$ found that maintaining apical patency during routine endodontic treatment was not associated with an increased incidence of postoperative pain in adult patients. In the present study, all canals obtained apical patency and they were instrumented with the same standard movement hybridization technique.

Many studies differ on the conduct of promoting the cleaning and eradication of microorganisms in single and multiple consultations, and many professionals attribute postoperative pain to the choice of one of the procedures. In the present study, the irrigating solution used by professionals was $2 \%$ chlorhexidine gel, whereas in the study by Onay et al., ${ }^{13}$ the irrigating solutions used were sodium hypochlorite, chlorhexidine, and EDTA + chlorhexidine, and there were no statistically significant differences in the incidence of postoperative pain according to the irrigating solutions used during treatment. In the study by Almeida et al., ${ }^{20}$ carried out in single consultations, the researchers compared two irrigating solutions in terms of postoperative pain: $5.25 \%$ sodium hypochlorite $(\mathrm{NaOCl})$ and $2 \%$ chlorhexidine gel. To assess postoperative pain, a questionnaire of pain intensity scale was used in 24, 48, 72 hours, and 7 days. No patient reported severe pain in the stages, $3 \%$ of patients $(2 / 63$ in each group) reported moderate pain after 24 hours, and no patient reported pain beyond 24 hours, regardless of the irritant used. Mild pain was more frequent, but decreased rapidly (reported by $19 \%$ [12/63] of patients in the $\mathrm{NaOCl}$ group and $16 \%[10 / 63]$ in the 24-hour chlorhexidine group, by $10 \%[6 / 63]$ in the $\mathrm{NaOCl}$ group and $11 \%$ [7/63] in the chlorhexidine group at 48 hours, by $3 \%$ [2/63] in both groups at 72 hours, and by $2 \%[1 / 63]$ in both groups at 7 days).

In the present study, the instrumentation technique was the hybridization of movements, the instrumentation limit was $1 \mathrm{~mm}$ beyond the foramen for dead pulp, and the filling technique used was hydraulic compression, single cone. In the study by Gambarini et al., ${ }^{21}$ the purpose was to evaluate and compare postoperative pain using three different rotary instrumentation techniques: crown-down rotary instrumentation technique using TF instruments (SybronEndo, Orange, CA), reciprocating instrumentation technique with a single instrument using the WaveOne instrument (Maillefer Dentsply, Ballaigues, $\mathrm{CH}$ ), and new instrumentation technique (TF Adaptive, SybronEndo, Orange, CA) using a single movement and combining reciprocating instrumentation and continuous rotary instrumentation. All techniques were performed following the manufacturer's instructions, and all root canals were treated, cleaned, and filled in single consultations by the same operator. Postoperative pain was assessed for three days using a visual analog scale. The results using the visual analog scale showed statistically significant differences between TF and TF Adaptive. When the patients' experience of severe pain was evaluated, the incidence of symptoms was significantly higher with the WaveOne technique.

\section{Conclusion}

After analyzing the medical records of patients subjected to endodontic treatments at the Endodontics Center, it was observed that the occurrence of postoperative pain was around 30\% in both single and multiple sessions, and it was considered relevant for the dental clinic. Pulp condition affected postoperative pain, and the presence of pain was associated with patients who underwent endodontics on teeth with live pulp. In addition, patients who had edema were more likely to have postoperative pain.

This result may be due to the current evolution of rotary and reciprocating systems that, even with new irrigation technologies and techniques, end up producing debris that can be taken out of the periapex, and mechanical movement can also cause pain.

\section{ACKnOWLedgments}

\section{Authorship Declaration}

Author contributions: The authors declare that all authors have contributed significantly and that all authors agree with the manuscript.

Fornari B worked in structuring the article, designeding the method, preparation of database, and analysis of results. Solda $C$ worked in project design, in data collection, and entering the database. Rigo $\mathrm{L}$ worked in discussion of the methodology, and in the statistical analysis. Rigo $L$ worked in review of the English language and the final wording of the article.

\section{ORCID}

Lilian Rigo @ http://orcid.org/0000-0003-3725-3047

\section{References}

1. Hameed AHE, Elmubarak BDS, Abu-bakr HN, et al. Postoperative pain in multiple-visit and single-visit root canal treatment. J Endod 2010;36(1):36-39. DOI: 10.1016/j.joen.2009.09.003.

2. Hepsenoglu Y, Eyuboglu TF, Ozcan M. Postoperative pain intensity after single- versus two-visit nonsurgical endodontic retreatment: a randomized clinical trial. J Endod 2018;44(9):1339-1346. DOI: 10.1016/j.joen.2018.05.017.

3. Pinheiro MLP, Lopes-Rocha R, Andrade ED. Periapical injection of betamethasone to control postoperative pain in emergency endodontic care - a randomized double blind clinical trial. Braz J Oral Sci 2017;15(3):209-214. DOI: 10.20396/bjos.v15i3.8649983

4. Ince B, Ercan E, Dalli M, et al. Incidence of postoperative pain after single- and multi-visit endodontic treatment in teeth with vital and non-vital pulp. Eur J Dent 2009;3(4):273-279. PMID: 19826598, PMCID: PMC2761157.

5. Abusrewil S, Alshanta OA, Albashaireh K, et al. Detection, treatment and prevention of endodontic biofilm infections: what's new in 2020? Crit Rev Microbiol 2020;46(2):194-212. DOI: 10.1080/1040841X.2020.1739622.

6. Silva EJ, Sa L, Belladonna FG, et al. Reciprocating versus rotary systems for root filling removal: assessment of the apically extruded material. J Endod 2014;40:2077-2080. DOI: 10.1016/j.joen.2014. 09.009

7. Kherlakian D, Cunha RS, Ehrhardt IC, et al. Comparison of the incidence of postoperative pain after using 2 reciprocating systems and a continuous rotary system: a prospective randomized clinical trial. J Endod 2016; 42(2):171-176. DOI: 10.1016/j.joen.2015. 10.011.

8. Tavares SG, Fontana CE, Martin AS, et al. In vivo evaluation of painful symptomatology after endodontic treatment performed 
using two different irrigation needle insertion depths. Eur J Dent 2020;14(2):274-280. DOI: 10.1055/s-0040-1709930.

9. Mostafa MEHAA, El-Shrief YAl, Anous WIO, et al. Postoperative pain following endodontic irrigation using $1.3 \%$ versus $5.25 \%$ sodium hypochlorite in mandibular molars with necrotic pulps: a randomized double-blind clinical trial. Int Endod J 2020;53(2):154-166. DOI: 10.1111/iej.13222.

10. Segura-Egea JJ, Cisneros-Cabello R, Llamas-Carreras JM, et al. Pain associated with root canal treatment. Int Endod J 2009;42(7):614-620. DOI: 10.1111/j.1365-2591.2009.01562.x.

11. Kirchhoff AL, Fariniuk LF, Mello I. Apical extrusion of debris in flat-oval root canals after using different instrumentation systems. J Endod 2015;41(2):237-241. DOI: 10.1016/j.joen.2014.09.023.

12. Bürklein S, Benten $S$, SchäferE. Quantitative evaluation of apically extruded debris with different single-file systems: Reciproc, F360 and OneShape versus Mtwo. Int Endod J 2014;47(5):405-409. DOI: 10.1111/iej.12161.

13. Onay EO, Ungor M, Yazici AC. The evaluation of endodontic flareups and their relationship to various risk factors. BMC Oral Health 2015;15(1):142. DOI: 10.1186/s12903-015-0135-2.

14. Baker NE, Liewehr FR, Buxton TB, et al. Antibacterial efficacy of calcium hydroxide, iodine potassium iodide, betadine, and betadine scrub with and without surfactant against E faecalis in vitro. Oral Surg Oral Med Oral Pathol Oral Radiol Endod 2004;98(3):359-364. DOI: 10.1016/j.tripleo.2004.04.023.
15. Direnzo A, Gresla T, Johnson BR, et al. Postoperative pain after 1- and 2-visit root canal therapy. Oral Surg Oral Med Oral Pathol Oral Radiol Endod 2002;93(5):605-610. DOI: 10.1067/moe.2002.121900.

16. Rigo L, Petrini I, Lodi, L. Postoperative pain in endodontic treatment in one session or multiple. Int J Dent 2012;11(1):29-37.

17. Iqbal $M$, Kurtz E, Kohli M. Incidence and factors related to flare-ups in a graduate endodontic programme. Int Endod J 2008;42(10):99-104. DOI: 10.1111/j.1365-2591.2008.01461.x.

18. Yaylali IE, Demirci GK, Kurnaz S, et al. Does maintaining apical patency during instrumentation increase postoperative pain or flare-up rate after nonsurgical root canal treatment? A systematic review of randomized controlled trials. J Endod 2018;44(8):1228-1236. DOI: 10.1016/j.joen.2018.05.002.

19. Abdulrab S, Rodrigues JC, Al-Maweri SA, et al. Effect of apical patency on postoperative pain: a meta-analysis. J Endod 2018;44(10):14671473. DOI: 10.1016/j.joen.2018.07.011.

20. Almeida G, Marques E, De Martin AS, et al. Influence of irrigating solution on postoperative pain following single-visit endodontic treatment: randomized clinical trial. JCDA 2012;78(84):1-6. PMID: 22985896.

21. Gambarini G, Testarelli L, De Luca M, et al. The influence of three different instrumentation techniques on the incidence of postoperative pain after endodontic treatment. Ann Stomatol 2013;4(1):152-155. DOI: 10.11138/ads.0152. 